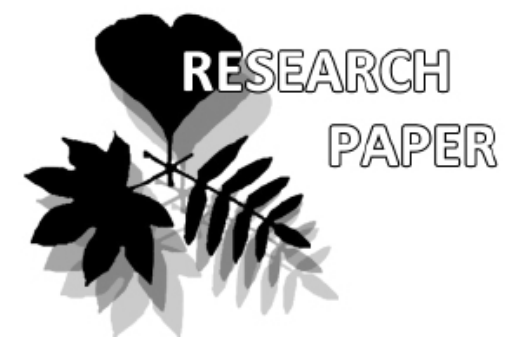

Tatiana V. Chernenkova ${ }^{1 *}$

e-mail: chernenkova50@mail.ru

Mikhail Yu. Puzachenko²

e-mail:puzak@bk.ru

Olga V. Morozova ${ }^{2}$

e-mail: olvasmor@mail.ru

Galina N. Ogureeva ${ }^{3}$

e-mail: ogur02@yandex.ru

Roman G. Kuperman ${ }^{4}$

roman.g.kuperman.civ@mail.mil

\section{${ }^{1}$ Centre for Forest Ecology and Produc- tion, RAS, Moscow, Russia \\ ${ }^{2}$ Institute of Geography, RAS, Moscow, Russia \\ ${ }^{3}$ M.V. Lomonosov Moscow State Univer- sity, Moscow, 119991, Russia \\ ${ }^{5}$ Edgewood Chemical Biological Center, Aberdeen Proving Ground, MD, USA}

* author for correspondence

Manuscript received: 20.03.2015

Review completed: 29.03.2015

Accepted for publication: 01.04.2015

Published online: 19.05.2015

\title{
An approach for mapping Northern Fennoscandian forests at different scales
}

\author{
Tatiana V. Chernenkova ${ }^{1 *}$, Mikhail Yu. Puzachenko², \\ Olga V. Morozova ${ }^{2}$, Galina N. Ogureeva ${ }^{3}$ \& Roman G. Kuperman ${ }^{4}$
}

\begin{abstract}
A B S T R A C T
Assessment of the diversity of forests throughout their distribution range poses a major challenge for researches because it involves a significant effort of collecting and analyzing heterogeneous information. Our approach to mapping and analyzing the present-day spatial diversity of forests in European Russia was based on a multi-parametric analysis of the chart community characteristics obtained by ground surveys, remote sensing data, and morphometric parameters of the relief. The main steps of methodology were: a) ground-based in a test region selected to represent the main zonal divisions of forest cover, b) development of uniform forest classification, c) mapping at local (large) scale, d) mapping at regional (small) scales. Forest classification was based on the relative availability of ecological and morphological groups of plant species in syntaxa and included seven hierarchical units. Two types of forest cover maps for Northern Fennoscandia are presented as examples at regional and local scales.

Ke ywords : dominant classification, boreal forest, cartographic modeling, statistical methods, GIS, hierarchical legend
\end{abstract}

\section{P E 3 Ю M E}

Черненькова Т.В., Пузаченко М.Ю., Морозова О.В., Огуреева Г.Н., Куперман Р.Г. ПодхоА к картографированию месов северной Фенноскандии в разных масштабах. Оценка разнообразия Аесов на протяжении их ареала представцяет собой большую проблему, которая требует приложения значительных усилий по сбору и анализу разнородного материала. Представ ен подход Аля картографирования и оценки современного пространственного разнообразия Аесов европейской части России в разАичных масштабах. Он основан на многопараметрическом анализе характеристик растительных сообществ, полученных при наземных обследованиях, Аанных дистанционного зондирования (ААЗ $)$ и морфометрических параметрах рельефа. Основными этапами нашей методологии явАяются: а) наземные исследования в тестовой области в соответствии с основными зональными полразделениями месного покрова, б) разработка единой классификации месов, в) картографирование на цокальном уровне в крупном масштабе, г) картографирование на региональном уровне в мелком масштабе. Аесная классификация основана на учете экологических и морфологических групп видов растений в синтаксонах и включает семь иерархических единиц: тип растительности, подтип растительности, кАасс формаций, формация, класс ассоциаций, группа ассоциаций и ассоциация. В качестве примера представ ены два типа карт цесного покрова Аля $\Lambda$ Окального и регионального уровня северной части Фенноскандии.

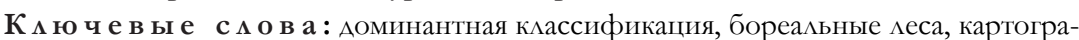
фическое моделирование, статистические методы, ГИС, Аистанционная информация

No m e n c la t u r e : Cherepanov (1995) for vascular plants, Ignatov et al. (2006) for mosses, Konstantinova \& Bakalin (2009) for liverworts, Santesson et al. (2004) for lichens.

\section{INTRODUCTION}

Exploration of the spatial differentiations of boreal forests at multiple structural levels is among pressing research needs for the vegetation science. Meaningful mapping of vegetation cover can reveal ecogeographic patterns in forest distributions and their regional specificity, as well as the role of anthropogenic factors in the formation of contemporary biodiversity.
Numerous survey maps have been developed for the European part of Russia. These maps were created using a variety of methodological approaches and principles for preparing the legends. Specific information on the Eastern-European forests can be found at regional and general maps: "Map of the vegetation of European part of the former USSR and the Transcaucasia" (Lavrenko 1976); "Map of Reconstructed vegetation of Central and Eastern Europe” (Gribova \& Neuhäusl 1989); "Map 'Ve- 
getation of the USSR" (Belov et al. 1990) and "Map of the Natural vegetation of Europe" (Bohn et al. 2004). These maps provide information on restored, or potential vegetation cover, and other important background scientific information. However, they may contain outdated information, including plant communities composition representative of the 1960-1970s, and do not accurately depict the present condition of the vegetation.

A more accurate representation of the spatial distribution of contemporary forest cover can be achieved by means of digital maps based on remote sensing information. One of the first successful products of digital cartography was the map "Vegetation of Russia" (scale $1: 7,500,000)$ that was developed on the basis of satellite images SPOT-Vegetation with a spatial resolution of $250 \mathrm{~m}$ (Bartalev et al. 2003, 2011). However, the legend of this map lacks the necessary details and provides only general information on the composition of present-day forests. Only large formation groups, such as Evergreen Dark Needle-leaf Forest, Evergreen Light Needle-leaf Forest, Deciduous Broadleaf Forest, Needleleaf with Broadleaf Forest, Mixed Forest, Broadleaf with Needleleaf Forest, Deciduous Needle-leaf Forest, and Sparse Deciduous Needle-leaf Forest, can be distinguished on this map. Notwithstanding these limitations, this map very accurately depicts the current forestland cover, possible disturbances (e.g., burned areas in the last few years), thus represents a phase in operative mapping on a small scale required for forest monitoring.

Recently, a mapping method that is based on combination of remote sensing and ground-based sources of information has gained a widespread acceptance (McRober 2006, Tomppo et al. 2008, Puzachenko \& Chernenkova 2012, Kravtzova 2013, Puzachenko et al. 2014, Roelofsen et al. 2014). This method is commonly used to create regional and local maps. However, cartographic information on the current vegetation and on the diversity of forest cover is not available for most regions of the European part of Russia. Another problem is the lack of uniform classification for the Russian forests, which would allow to compare the forests from different part of their range and evaluate their diversity (Zaugolnova \& Morozova 2006). Study of the vegetation diversity at either large or small geographical scales is closely related to classification of plant communities (Walker 1999, Rodwel et al. 2002, Rysin \& Savelyeva 2007). Finally, development of a common methodology is needed to identify the factors that affect the forest diversity at different scales.

To fill the current methodological gaps, a team of experts from different research centers (Center for Productivity of Forests and Institute of Geography of the Russian Academy of sciences, Geography Department of Moscow State University) is assessing current plant diversity of the Eastern European boreal and hemi-boreal forests, including their regional specificity and distribution. An effort to map the forest vegetation for the Eastern European part of Russia at different scales was among the objectives of this team. This paper discusses the methodology for detecting a spatial diversity of forests by using remote sensing technology in order to create maps at large and small scales using northern part of Fennoscandia as an example.

\section{METHODS AND DATA}

Present study developed and utilized methodology and methods for cartographic modeling of vegetation at different spatial scales (Fig. 1). It was based on multiparametric analysis of the characteristics of plant communities obtained by ground surveys, remote sensing data (RSD), and morphometric parameters of the ground surface. We conducted field survey within test area and stored geobotanical features into database for further analyses in the form of classification units to create maps, design a map legend, and assess a forest diversity. These data can be utilized in large- or medium-scale maps at scales ranging between 1:50,000 and 1: 200,000 to show a current diversity of vegetation. The results of the local assessment of the vegetation surveys were used to map the structure of the vegetation cover at the regional level and for the creation of small-scale map within a corresponding zonal division.

\section{Study area and field data}

The large scale study area is located in the central part of the Kola Peninsula $\left(67^{\circ} 50^{\prime} \mathrm{N}, 32^{\circ} 35^{\prime} \mathrm{E}\right)$ and belongs to the northern taiga subzone (Fig. 2). Total area of this model territory is approximately $8400 \mathrm{~km}^{2}$. It covers the Lake Imandra basin, which includes the territory of the Lapland Nature Reserve and a large part of Khibiny mountains area with mining and ore enrichment plants (Kola Mining and Metallurgical Company). The territory is characterized by a complex, rugged relief, the altitudes range from 100 to $1200 \mathrm{~m}$ a.s.l.

Geobotanical data of plant communities were obtained on sampling plots (size $20 \times 20 \mathrm{~m}$ ) with exact geographical positions determined by GPS. The sampling plots were selected on basis of preliminary analysis of RSD, digital elevation model (DEM), topographic and forest inventory maps and to be representative of regional diversity of forest communities and their anthropogenic modifications. In addition, non-forest vegetation was examined. As many as 500 geobotanical plots were sampled in the model study area. These plots were selected to be representative of the majority of vegetation diversity in the region. Different types of land cover and water bodies that are not represented in the sample plots were determined from all available spatial information (280 points).

At small-scale $(1: 2,000,000)$, the vegetation map is represented by a fragment of Fennoscandia region with total area about $540,000 \mathrm{~km}^{2}$, which includes North Scandinavia and the Kola Peninsula.

\section{Forest classification}

A uniform eco-phytocoenological forest classification was developed to assess the typological diversity of forests. This forest classification was based on the relative presence of ecological and morphological groups of plant species in syntaxa (Zaugolnova \& Morozova 2006, Chernenkova et al. 2012). A hierarchical classification included the following units: vegetation type, vegetation subtype, formation class 


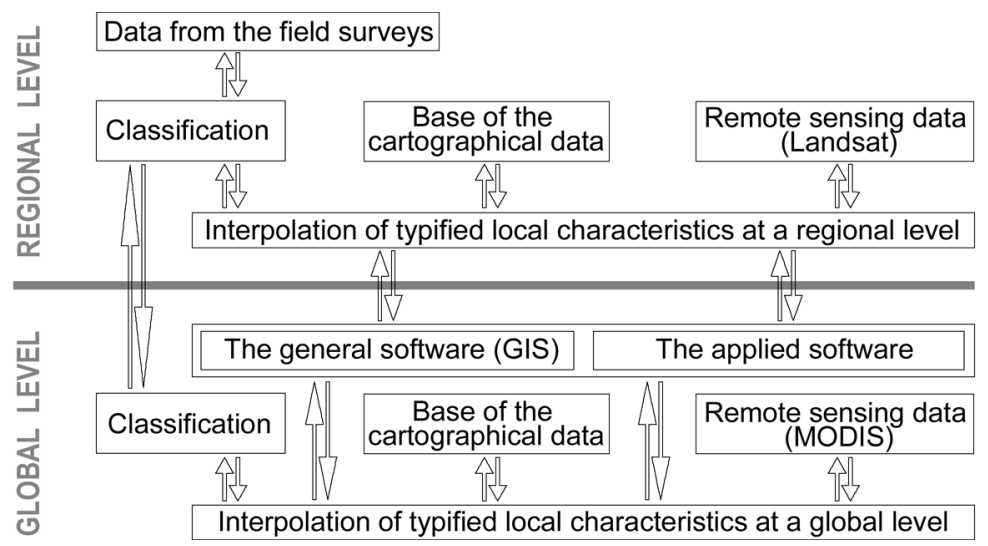

shed information on the vegetation of different regions (Bohn at al. 2004, Saucier et al. 2013). At large and medium scales (from 1:50,000 to $1: 200,000)$, the main mappable units for forest vegetation were groups of association for zonal communities, and classes of association for azonal vegetation types (e.g., wetlands, meadows). Units of lower level were identified on the basis of diagnostic or indicator species that revealed the regional specificity of communities. Association class and formation class were the base units for mapping at a small scale.

Figure 1 The main stages of forest mapping at different scales

(group), formation, association class, association group, and association. Vegetation type was based on a biomorphic type; subtype was based on floristic and phylogenetic commonality (Sochava 1964, Lavrenko 1968). Formation class was determined on the basis of the predominant life form of tree species (e.g., dark coniferous, light coniferous forests). Formation group was based on genera of the dominant tree species (e.g., spruce, pine forests). Formation was determined by predominant species of tree stand. Association class was characterized by both composition of tree stand edificators and composition of the predominant ecological and morphological groups of understory species. The association group was characterized by dominant species groups at main layers of forest community.

The development of forest classification involved both the analyses of field data and the review of available publi-

\section{Vegetation mapping at large scale}

Each sample plot was correlated with association group for forests communities and association class for non-forest vegetation. Other land cover classes (e.g., water bodies, settlements) were determined from topographic maps and RSD.

The basis for large-scale interpolation of vegetation classes were Landsat 5, 7, 8 and relief data. Landsat data for analysis are presented by images for the last 10-15 years and for different seasons. Both the brightness values of channels as well as the derived 24 indices (NDVI, VI, RVI, QRVI, DVI, TSA, R/G, TVI, GNDVI, TNDVI, NDWI, NDSI, SAVI and etc.) were used in analysis. Model area covers several Landsat scenes that required mosaicking images. This was accomplished by using ErdasImagine mosaicking with histogram correction. As a result, seamless mosaics of Landsat images were obtained from May to October.

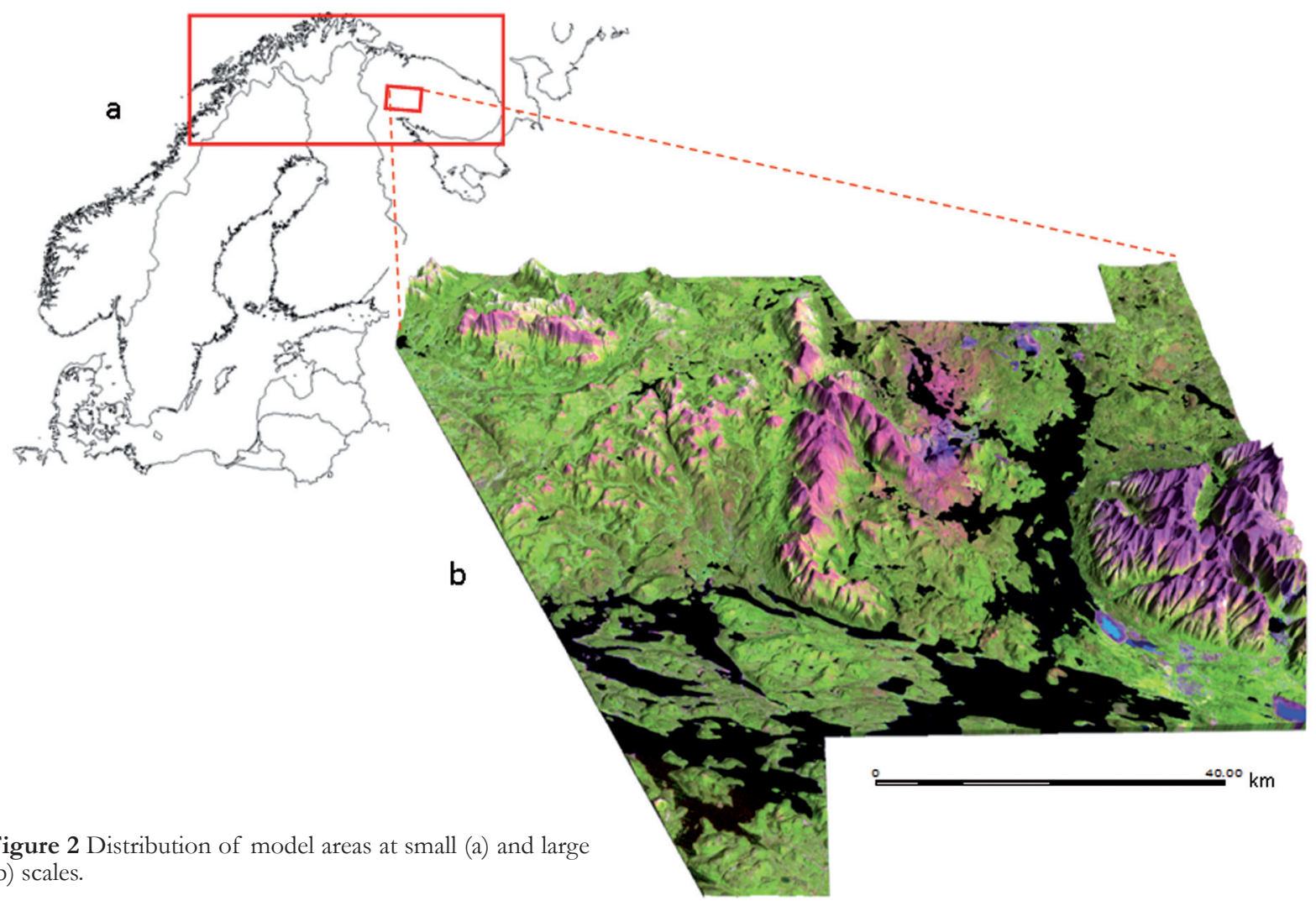

(b) scales. 


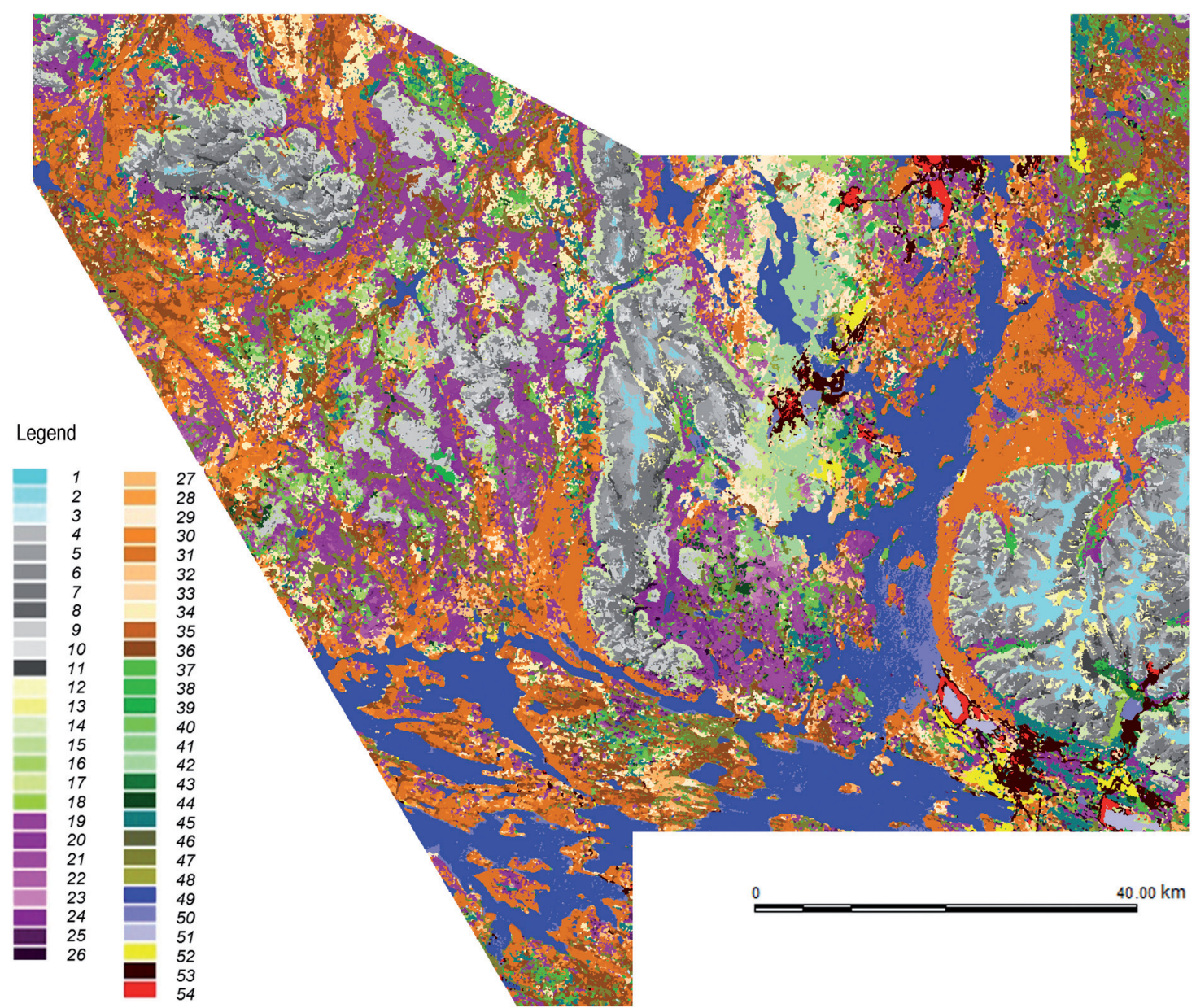

Figure 3 Vegetation map of model area at large scale, for legend see Table 1

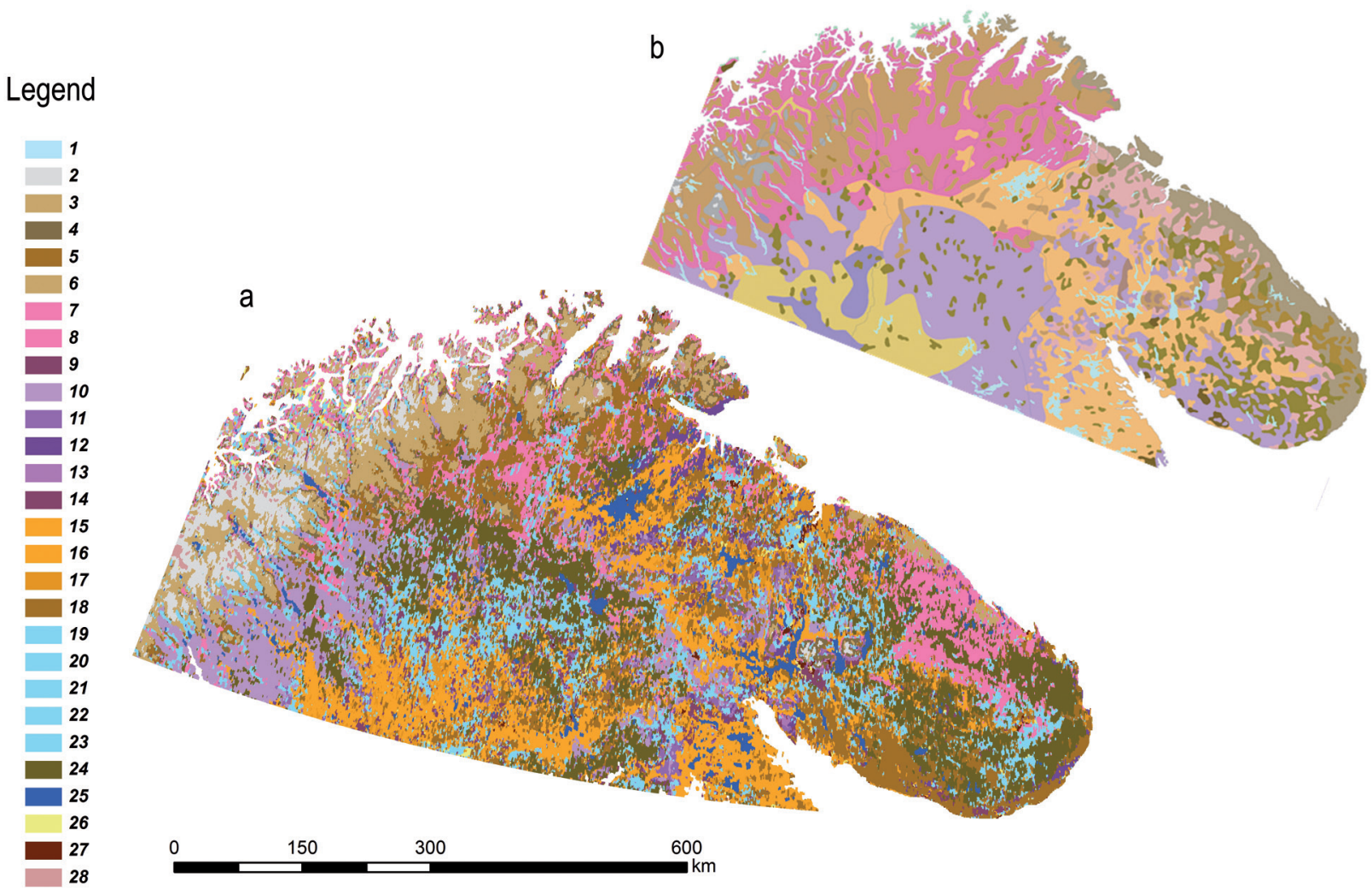

Figure 4 Vegetation map of the Northern Fennoscandia at small scale: a - the present effort version of the vegetation map for association classes, for legend see Table 1; b - fragment of the Natural vegetation European Map (Bohn et al. 2000) 
Table 1. Classification scheme for vegetation of the northern part of Fennoscandia. Secondary vegetation types are marked with asterisks; numbers in arabic mark association classes on the small scale maps and association groups - on large scale maps

\begin{tabular}{|c|c|c|c|}
\hline $\begin{array}{l}\text { Vegeta- } \\
\text { tion type }\end{array}$ & $\begin{array}{l}\text { Formation } \\
\text { class }\end{array}$ & Association class & Association group \\
\hline Nival area & Nival area & 1. Nival area & 1. Nival area \\
\hline \multirow[t]{12}{*}{ Tundra } & \multirow[t]{2}{*}{$\begin{array}{l}\text { Stone golsty } \\
\text { barrens }\end{array}$} & \multirow{2}{*}{$\begin{array}{l}\text { 2. Barren landscapes with very } \\
\text { sparse, very low plant cover }(<10 \%) \\
\text { with scattered crustose and fruticose } \\
\text { lichens, dwarf shrubs and herbs }\end{array}$} & $\begin{array}{l}\text { 2. Stone golsty barrens and sparse epilitic/crustose lichens (Rhizocarpon } \\
\text { geographicum, Umbilicaria spp., Arctoparmelia centrifuga) tundra }\end{array}$ \\
\hline & & & $\begin{array}{l}\text { 3. Sparse fragments of dwarf shrubs (Silene acaulis, Empetrum hermaphrodi- } \\
\text { tum), grass (Juncus trifidus, Festuca ovina, Carex bigelowii) and lichens (Flavo- } \\
\text { cetraria nivalis, Alectoria ochroleuca, Cetraria islandica) }\end{array}$ \\
\hline & $\begin{array}{l}\text { Sparse } \\
\text { tundra with } \\
\text { epilithic } \\
\text { lichen com- } \\
\text { munities }\end{array}$ & $\begin{array}{l}\text { 3. Sparse tundra vegetation } \\
(10-30 \%) \text { of flat tops and crests. } \\
\text { Prostrate lichens, mosses-lichen } \\
\text { and dwarf shrub-lichen groups }\end{array}$ & $\begin{array}{l}\text { 4. Sparse dwarf shrub (Empetrum hermaphroditum, Saxifraga oppositifolia, } \\
\text { Dryas octopetala, Loiseleuria procumbens, Diapensia lapponica) with mosses } \\
\text { (Racomitrium lanuginosum) and lichens (Flavocetraria nivalis, Alectoria ochroleuca, } \\
\text { Cetraria islandica) tundra }\end{array}$ \\
\hline & \multirow{3}{*}{$\begin{array}{l}\text { Dense } \\
\text { lichen } \\
\text { tundras }\end{array}$} & \multirow{3}{*}{$\begin{array}{l}\text { 4. Dense }(>30 \%) \text { lichen vegeta- } \\
\text { tion on the tops and slopes of } \\
\text { mountains with dwarf shrub-moss } \\
\text { complexes }\end{array}$} & $\begin{array}{l}\text { 5. Lichen-rich (Cladonia stellaris, C. arbuscula, Alectoria ochroleuca, Cetraria } \\
\text { islandica, Flavocetraria nivalis) tundra }\end{array}$ \\
\hline & & & $\begin{array}{l}\text { 6. Dwarf shrub (Empetrum hermaphroditum, Arctous alpina, Betula nana, } \\
\text { Carex bigelowii) with lichens (Cladonia stellaris, C. arbuscula, Flavocetraria } \\
\text { nivalis, Cetraria islandica) tundra }\end{array}$ \\
\hline & & & $\begin{array}{l}\text { 7. Dwarf shrub (Empetrum hermaphroditum, Vaccinium myrtillus, Betula nana) } \\
\text { with mosses (Pleurozium schreberi, Ptilidium ciliare, Barbilophozia spp., Poblia } \\
\text { nutans) and lichens (Cladonia stellaris, Cladonia arbuscula, Cetraria islandica, } \\
\text { Orthocaulis spp.) tundra }\end{array}$ \\
\hline & \multirow{4}{*}{$\begin{array}{l}\text { Dense } \\
\text { dwarf } \\
\text { shrub, } \\
\text { shrub } \\
\text { and partly } \\
\text { mosses } \\
\text { tundras }\end{array}$} & \multirow[t]{4}{*}{$\begin{array}{l}\text { 5. Dense }(>30 \%) \text { vegetation on } \\
\text { the tops and slopes of mountains } \\
\text { with dwarf shrubs and shrubs }\end{array}$} & $\begin{array}{l}\text { 8. Dwarf shrub (Empetrum hermaphroditum, Vaccinium myrtillus, Dryas octo- } \\
\text { petala, Loiseleuria procumbens), shrub (Salix spp., Betula nana), sedge (Carex } \\
\text { bigelowii) and partly with mosses (Pleurozium schreberi, Racomitrium lanugi- } \\
\text { nosum) tundra }\end{array}$ \\
\hline & & & $\begin{array}{l}\text { 9*. Dwarf shrub (Betula nana, Vaccinium uliginosum, Vaccinium vitis-idaea), } \\
\text { herb (Avenella flexuosa, Deschampsia caespitosa, Chamaenerion angustifolium) } \\
\text { fragments of vegetation }\end{array}$ \\
\hline & & & $\begin{array}{l}\text { 10. Herb (Carex bigelowii, Juncus trifidus) with lichens (Umbilicaria spp., Stere- } \\
\text { ocaulon alpinum) tundra }\end{array}$ \\
\hline & & & $\begin{array}{l}\text { 11. Dense shrub (Betula nana, Salix lapponum, S. glauca), dwarf shrub } \\
\text { (Empetrum hermaphroditum, Vaccinium myrtillus) and partly with mosses (Hy- } \\
\text { locomium splendens, Pleurozium schreberi) meadow community }\end{array}$ \\
\hline & \multirow{2}{*}{\begin{tabular}{|l|} 
Herbs com- \\
munites in \\
depressions \\
and mead- \\
ow azonal \\
tundra's \\
complexes \\
\end{tabular}} & \multirow{2}{*}{$\begin{array}{l}\text { 6. Meadow complexes in valley of } \\
\text { streams and on moist gentle slopes } \\
\text { and dwarf-small herb groups }\end{array}$} & $\begin{array}{l}\text { 12. Small herb, grass (Anthoxanthum alpinum, Avenella flexuosa, Nardus } \\
\text { stricta) meadow }\end{array}$ \\
\hline & & & $\begin{array}{l}\text { 13. Willow (Salix phylicifolia, Salix lapponum, Salix glauca, Betula nana), } \\
\text { dwarf shrub (Empetrum hermaphroditum, Vaccinium myrtillus), herb (Saxifraga } \\
\text { stellaris, Oxyria digyna) with mosses tundra in depressions and meadow } \\
\text { complexes with herbs (Anthoxanthum alpinum, Geranium sylvaticum, Trollius } \\
\text { europaeus) }\end{array}$ \\
\hline \multirow[t]{5}{*}{$\begin{array}{l}\text { Forest- } \\
\text { tundra }\end{array}$} & \multirow{5}{*}{$\begin{array}{l}\text { Northeast } \\
\text { European } \\
\text { subalpine } \\
\text { prostrate } \\
\text { birch stands } \\
\text { (krum- } \\
\text { mholz) and } \\
\text { forest- } \\
\text { tundras }\end{array}$} & $\begin{array}{l}\text { 7. Subalpine prostrate birch wood- } \\
\text { lands (krummholz) (Betula pubescens } \\
\text { subsp. czerepanovii) partly with Picea } \\
\text { obovata and Pinus sylvestris, with } \\
\text { dwarf shrubs and lichens }\end{array}$ & $\begin{array}{l}\text { 14. Dwarf shrub (Empetrum hermaphroditum, Arctostaphylos wva-ursi, Betula } \\
\text { nana, Calluna vulgaris) with lichens (Flavocetraria nivalis, Cladonia stellaris, } \\
\text { C. arbuscula, Cetraria islandica); Dwarf shrub (Empetrum hermaphroditum, } \\
\text { Vaccinium myrtillus, V. vitis-idaea, Betula nana) with mosses (Pleurozium schre- } \\
\text { beri, Hylocomium splendens) and lichens (Cladonia stellaris, Cetraria islandica, } \\
\text { Flavocetraria nivalis) prostrate birch woodlands }\end{array}$ \\
\hline & & \multirow{3}{*}{$\begin{array}{l}\text { 8. Subalpine prostrate birch wood- } \\
\text { lands (krummholz) (Betula pubescens } \\
\text { subsp. czerepanovii) with Picea obo- } \\
\text { vata and Pinus sylvestris, with dwarf } \\
\text { shrubs and partly with lichens }\end{array}$} & $\begin{array}{l}\text { 15*. Dwarf shrub (Empetrum hermaphroditum, Arctostaphylos uva-ursi) pros- } \\
\text { trate birch woodlands }\end{array}$ \\
\hline & & & $\begin{array}{l}\text { 16*. Dwarf shrub (Empetrum hermaphroditum, Arctous alpina, Vaccinium myr- } \\
\text { tillus), herb (Carex bigellowii, Juncus trifidus, Avenella flexuosa) with crustose } \\
\text { lichens (Trapeliopsis granulosa, Cladonia deformis) prostrate birch woodlands }\end{array}$ \\
\hline & & & $\begin{array}{l}\text { 17*. Sparse dwarf shrub and mosses (Poblia nutans) prostrate birch wood- } \\
\text { lands }\end{array}$ \\
\hline & & $\begin{array}{l}\text { 9. Subalpine prostrate birch wood- } \\
\text { lands (krummholz) (Betula pubescens } \\
\text { subsp. czerepanovii) with Picea obovata } \\
\text { and Pinus sylvestris, with dwarf } \\
\text { shrubs and herbs }\end{array}$ & $\begin{array}{l}\text { 18. Dwarf shrub (Vaccinium myrtillus), herb (Chamaepericlymenum suecicum, } \\
\text { Chamaenerion angustifolium, Geranium sylvaticum) prostrate birch woodlands }\end{array}$ \\
\hline \multirow[t]{2}{*}{$\begin{array}{l}\text { Dark } \\
\text { coniferous } \\
\text { forest }\end{array}$} & \multirow[t]{2}{*}{$\begin{array}{l}\text { Northeast } \\
\text { European } \\
\text { open spruce } \\
\text { forests }\end{array}$} & \begin{tabular}{|l|} 
10. Spruce forests (Picea abies $\times$ \\
Picea obovata, P. obovata) with Pinus \\
sylvestris, Betula pubescens, B. pubescens \\
subsp. czerepanovii, with dwarf \\
shrubs, mosses and lichens
\end{tabular} & $\begin{array}{l}\text { 19. Dwarf shrub (Empetrum hermaphroditum, Vaccinium myrtillus, Ledum } \\
\text { palustre) with mosses (Pleurozium schreberi, Hylocomiun splendens) and lichens } \\
\text { (Cetraria islandica, Cladonia spp.) spruce forest }\end{array}$ \\
\hline & & $\begin{array}{l}\text { 11. Spruce forests (Picea abies } \times \\
\text { Picea obovata, P. obovata) with Pinus } \\
\text { sylvestris, Betula pubescens, B. pubescens } \\
\text { subsp. czerepanovii, with dwarf } \\
\text { shrubs, herbs and mosses }\end{array}$ & $\begin{array}{l}\text { 20. Dwarf shrub (Empetrum hermaphroditum, Vaccinium uliginosum, } V \text {. } \\
\text { myrtillus, V. vitis-idaea), herb (Chamaepericlymenum suecicum, Gymnocarpium } \\
\text { dryopteris, Avenella flexousa) with mosses (Pleurozium schreberi, Hylocomiun } \\
\text { splendens) spruce forest }\end{array}$ \\
\hline
\end{tabular}


Table 1. Continued

\begin{tabular}{|c|c|c|c|}
\hline $\begin{array}{l}\text { Vegeta- } \\
\text { tion type }\end{array}$ & $\begin{array}{l}\text { Formation } \\
\text { class }\end{array}$ & Association class & Association group \\
\hline \multirow[t]{6}{*}{$\begin{array}{l}\text { Dark } \\
\text { coniferous } \\
\text { forest }\end{array}$} & \multirow{6}{*}{$\begin{array}{l}\text { Northeast } \\
\text { European } \\
\text { open spruce } \\
\text { forests }\end{array}$} & \multirow{3}{*}{$\begin{array}{l}\text { 12. Spruce forests (Picea abies } \times \\
\text { Picea obovata, P. obovata) with Pinus } \\
\text { sylvestris, Betula pubescens, B. pubescens } \\
\text { subsp. czerepanovii, with dwarf } \\
\text { shrubs, partly with mosses and } \\
\text { lichens }\end{array}$} & $\begin{array}{l}\text { 21*. Dwarf shrub (Empetrum hermaphroditum, Vaccinium myrtillus), with liv- } \\
\text { erworts (Barbilophozia spp., Lophosia spp., Orthocaulis spp.) and partly with } \\
\text { lichens (Cetraria islandica, Cladonia spp.) spruce forest }\end{array}$ \\
\hline & & & $\begin{array}{l}\text { 22*. Dwarf shrub (Empetrum hermaphroditum, Vaccinium uliginosum, } V \text {. } \\
\text { myrtillus), herb (Avenella flexuosa) spruce forest }\end{array}$ \\
\hline & & & $\begin{array}{l}\text { 23*. Dwarf shrubs with crustose lichens (Trapeliopsis granulosa, Cladonia } \\
\text { deformis) spruce forest }\end{array}$ \\
\hline & & $\begin{array}{l}\text { 13. Spruce forests (Picea abies } \times \\
\text { Picea obovata, P. obovata) with Betula } \\
\text { pubescens, with herb and tall-forb }\end{array}$ & $\begin{array}{l}\text { 24. Herb (Chamaepericlymenum suecicum, Gymnocarpium dryopteris, Avenella } \\
\text { flexousa), tall-forb (Cicerbita alpina, Geranium sylvaticum) spruce forest }\end{array}$ \\
\hline & & \multirow[t]{2}{*}{$\begin{array}{l}\text { 14. Spruce forests (Picea obovata), } \\
\text { with herb and sedges, and peat } \\
\text { mosses }\end{array}$} & $\begin{array}{l}\text { 25. Dwarf shrub (Rubus chamaemorus, Vacciniun myrtillus, V. uliginosum, } \\
\text { Empetrum hermaphroditum) with peat mosses (Sphagnum girgensohnii) spruce } \\
\text { forest }\end{array}$ \\
\hline & & & $\begin{array}{l}\text { 26. Herb and sedges (Carex nigra, Carex rostrata, Equisetum palustre, Erio- } \\
\text { phorum vaginatum) with peat mosses (Sph. girgensohnii, Sph. russowii, Sph. } \\
\text { angustifolium) spruce forest }\end{array}$ \\
\hline \multirow{10}{*}{$\begin{array}{l}\text { North- } \\
\text { ern light } \\
\text { coniferous } \\
\text { forest }\end{array}$} & \multirow[t]{10}{*}{ Pine forests } & \multirow{3}{*}{$\begin{array}{l}\text { 15. North European open pine fo- } \\
\text { rests (Pinus sylvestris) with Betula pubes- } \\
\text { cens, with dwarf shrubs and lichens }\end{array}$} & 27. Lichens (Cladonia stellaris, C. arbuscula, C. rangiferina) open pine forest \\
\hline & & & $\begin{array}{l}\text { 28. Dwarf shrub (Calluna vulgaris, Arctous alpina, Vaccinium myrtillus, V. vi- } \\
\text { tis-idaea, Empetrum hermaphroditum) with lichens (Cladonia stellaris, C. arbus- } \\
\text { cula, C. rangiferina) open pine forest }\end{array}$ \\
\hline & & & $\begin{array}{l}\text { 29*. Dwarf shrub with crustose lichens (Trapeliopsis granulosa) open pine } \\
\text { forest }\end{array}$ \\
\hline & & \multirow[t]{2}{*}{$\begin{array}{l}\text { 16. North European open pine } \\
\text { forests (Pinus sylvestris) with Betula } \\
\text { pubescens, with dwarf shrubs and } \\
\text { mosses }\end{array}$} & $\begin{array}{l}\text { 30. Dwarf shrub (Vaccinium vitis-idaea, V. uliginosum, Empetrum berma- } \\
\text { phroditum) with mosses (Dicranum majus, Pleurozium schreberi, Polytricbum } \\
\text { juniperinum) and lichens (Cetraria islandica, Cladonia stellaris, C. arbuscula, C. } \\
\text { rangiferina) open pine forest }\end{array}$ \\
\hline & & & $\begin{array}{l}\text { 31. Dwarf shrub (Vaccinium myrtillus, V. vitis-idaea, Empetrum hermaphrodi- } \\
\text { tum, Ledum palustre, Betula nana) with mosses (Dicranum majus, Pleurozium } \\
\text { schreberi, Polytrichum juniperinum) open pine forest }\end{array}$ \\
\hline & & \multirow{3}{*}{$\begin{array}{l}\text { 17. North European open pine } \\
\text { forests (Pinus sylvestris) with Betula } \\
\text { pubescens, with dwarf shrubs and } \\
\text { partly with mosses }\end{array}$} & $\begin{array}{l}\text { 32*. Dwarf shrub (Vaccinium myrtillus, V. vitis-idaea, V. uliginosum, Calluna } \\
\text { vulgaris, Avenella flexuosa), partly with liverwort mosses (Barbilophozia spp., } \\
\text { Orthocaulis spp.) open pine forest }\end{array}$ \\
\hline & & & $\begin{array}{l}33^{*} \text {. Dwarf shrub (Ledum palustre, Vaccinium vitis-idaea, Calluna vulgaris) } \\
\text { with fragments of moss cover (Poblia nutans) open pine forest }\end{array}$ \\
\hline & & & $\begin{array}{l}\text { 34*. Dwarf shrub (Vaccinium myrtillus, Empetrum hermaphroditum, Calluna } \\
\text { vulgaris) with mosses (Polytrichum spp.) open pine forest }\end{array}$ \\
\hline & & \multirow[t]{2}{*}{$\begin{array}{l}\text { 18. Northeast European open } \\
\text { hygrophilous pine forests (Pinus } \\
\text { sylvestris) with herbs, sedges and } \\
\text { peat mosses }\end{array}$} & $\begin{array}{l}\text { 35. Dwarf shrub (Vaccinium uliginosum, Andromeda polifolia, Chamaedaphne calycu- } \\
\text { lata, Ledum palustre), herb and sedge (Carex spp., Eriophorum vaginatum, Molinia } \\
\text { coerulea, Baeothyon cespitosum) with peat mosses (Sph. magellanicum, Sph. russowii) } \\
\text { open pine forest }\end{array}$ \\
\hline & & & $\begin{array}{l}\text { 36. Herb (Molinia coerulea, Baeothyon cespitosum), sedge (Carex spp., Eriopho- } \\
\text { rum vaginatum) with peat mosses (Sph. magellanicum, Sph. russowii) open pine } \\
\text { forest }\end{array}$ \\
\hline \multirow[t]{7}{*}{$\begin{array}{l}\text { Northern } \\
\text { small- } \\
\text { leaved } \\
\text { forest }\end{array}$} & \multirow[t]{7}{*}{$\begin{array}{l}\text { Birch fo- } \\
\text { rests }\end{array}$} & $\begin{array}{l}\text { 19. Northeast European open } \\
\text { birch woodlands (Betula pubescens } \\
\text { subsp. czerepanovii), partly with Picea } \\
\text { obovata, Pinus sylvestris) with dwarf } \\
\text { shrubs and lichens }\end{array}$ & $\begin{array}{l}\text { 37. Dwarf shrub (Ledum palustre, Vaccinium myrtillus) with lichens (Cladonia } \\
\text { stellaris, C. arbuscula, C. rangiferina, C. cenotea, C. deformis) open birch wood- } \\
\text { land }\end{array}$ \\
\hline & & \multirow{2}{*}{$\begin{array}{l}\text { 20. Northeast European open birch } \\
\text { woodlands (Betula pubescens), partly } \\
\text { with Picea obovata and Pinus sylvestris, } \\
\text { with dwarf shrubs, herbs and green } \\
\text { mosses }\end{array}$} & $\begin{array}{l}\text { 38. Dwarf shrub (Empetrum hermaphroditum, Vaccinium uliginosum, V. myrtil- } \\
\text { lus, V. vitis-idaea) with mosses (Pleurozium schreberi, Hylocomiun splendens) } \\
\text { open birch woodland }\end{array}$ \\
\hline & & & $\begin{array}{l}\text { 39. Dwarf shrub (Empetrum hermaphroditum, Vaccinium uliginosum, V. myrtil- } \\
\text { lus, V. vitis-idaea), herb (Chamaepericlymenum suecicum, Avenella flexousa) with } \\
\text { mosses (Pleurozium schreberi, Hylocomiun splendens) open birch woodland }\end{array}$ \\
\hline & & \multirow{3}{*}{$\begin{array}{l}\text { 21. Northeast European open } \\
\text { birch woodlands (Betula pubescens), } \\
\text { partly with Picea obovata and Pinus } \\
\text { sylvestris, with dwarf shrubs }\end{array}$} & $\begin{array}{l}\text { 40*.Dwarf shrub (Vaccinium myrtillus, Empetrum bermaphroditum), herb } \\
\text { (Avenella flexuosa, Chamaepericlymenum suecicum) open birch woodland }\end{array}$ \\
\hline & & & $\begin{array}{l}41 * \text {. Dwarf shrub with crustose lichens (Trapeliopsis granulosa) open birch } \\
\text { partly with willow woodland }\end{array}$ \\
\hline & & & $\begin{array}{l}42 * \text { Sparse dwarf shrubs with mosses (Poblia nutans) open birch partly } \\
\text { with willow woodland }\end{array}$ \\
\hline & & $\begin{array}{l}\text { 22. Northeast European birch } \\
\text { woodlands (Betula pubescens) with } \\
\text { Picea obovata with dwarf shrubs, } \\
\text { with tall herbs partly with mosses }\end{array}$ & $\begin{array}{l}\text { 43. Herb (Chamaenerion angustifolium, Geranium sylvaticum, Equisetum palustre), } \\
\text { dwarf shrub }(V . \text { myrtillus, } V \text {. vitis-idaea), tall herb (Chamaenerion angustifo- } \\
\text { lium, Geranium sylvatica, Equisetum palustre) partly with mosses (Poblia nutans, } \\
\text { Polytrichum spp.) open birch woodland }\end{array}$ \\
\hline
\end{tabular}


Table 1. Continued

\begin{tabular}{|c|c|c|c|}
\hline $\begin{array}{l}\text { Vegeta- } \\
\text { tion type }\end{array}$ & $\begin{array}{l}\text { Formation } \\
\text { class }\end{array}$ & Association class & Association group \\
\hline \multirow{2}{*}{$\begin{array}{l}\text { Northern } \\
\text { small- } \\
\text { leaved } \\
\text { forest }\end{array}$} & \multirow[t]{2}{*}{$\begin{array}{l}\text { Birch fo- } \\
\text { rests }\end{array}$} & \multirow{2}{*}{$\begin{array}{l}\text { 23. Northeast European open hyg- } \\
\text { rophilous birch woodlands (Betula pu- } \\
\text { bescens) with Picea obovata, with dwarf } \\
\text { shrubs, herbs, sedges and mosses }\end{array}$} & $\begin{array}{l}\text { 44. Dwarf shrub (Betula nana, Rubus chamaemorus, Ledum palustre), herb } \\
\text { (Equisetum palustre) with peat mosses (Sphagnum girgensobnii) open birch } \\
\text { woodland }\end{array}$ \\
\hline & & & $\begin{array}{l}\text { 45*. Dwarf shrub (Vaccinium myrtillus, Empetrum hermaphroditum), herb } \\
\text { (Avenella flexuosa, Chamaepericlymenum suecicum) aspen woodland }\end{array}$ \\
\hline \multirow[t]{3}{*}{ Mire } & \multirow[t]{3}{*}{ Mires } & \multirow[t]{3}{*}{ 24. Bogs } & $\begin{array}{l}\text { 46. Herb (Eriophorum vaginatum, Carex rotundata, C. rariflora) with peat } \\
\text { mosses bog (Sph. compactum, Sph. fuscum) }\end{array}$ \\
\hline & & & $\begin{array}{l}\text { 47. Dwarf shrub (Ledum palustre, Rubus chamaemorus, Andromeda polifolia) } \\
\text { with peat mosses (Sph. fuscum, Sph. russowii)) bog }\end{array}$ \\
\hline & & & 48. Dwarf shrub, herb with peat mosses (Sph. fuscum) aapa-complex \\
\hline \multicolumn{4}{|c|}{ Not vegetation categories of legend } \\
\hline & & \multirow[t]{3}{*}{ 25. Lakes and rivers } & 49. Lakes and rivers \\
\hline & & & 50. Polluted waters \\
\hline & & & 51. Waters for sedimentation \\
\hline & & 26. Farmlands & 52. Farmlands \\
\hline & & 27. Settlements & 53. Settlements \\
\hline & & 28. Mining dumps and open pits & 54. Mining dumps and open pits \\
\hline
\end{tabular}

The DEM of model area was obtained from topographic maps $(1: 50,000)$ by contour lines digitizing, followed by non-linear interpolation in ErdasImagine. The DEM cell size was determined to be $60 \mathrm{~m}$, which corresponds to the scale of topographic maps and multiple to Landsat scenes cell size. Morphometric characteristics of the relief forms were calculated for different sizes of the moving window, thus allowing to take into account the relief structure (Puzachenko et al. 2002). The seven levels of relief structures were determined with linear sizes from 180 to $3300 \mathrm{~m}$. Morphological characteristics of relief were estimated in program complex Environment for visualizing images (ENVI).

For interpolation of vegetation classes, the sample plots were merged with RSD and DEM in GIS. Interpolation of vegetation classes were based on the stepwise discriminant analysis (Kim et al. 1989, Puzachenko 2004, Kozlov et al. 2008). Statistically significant relationships among vegetation classes and "external" variables (RSD, DEM) were used for interpolation of the investigated classes for the entire model area. Furthermore, it allowed us to understand better spatial variation in vegetation classes in relation to various factors (characteristics of the terrain, natural and anthropogenic disturbances, etc.). Assessments of interpolation significance were based on probabilities of class attribution for each grid cells and relative quality of the discriminant analysis. Single cells were removed from grid of interpolated classes and replaced by the neighboring cells. After that, grid of vegetation classes in association group rank was converted to vector format for editing and making a final map.

\section{Vegetation mapping at small scale}

The small-scale vegetation maps were obtained on the basis of interpolation of the large-scale vegetation maps. The external variables for interpolating of regional vegetation maps to small-scale maps were MODIS multispectral data MCD43B4 (NASA LP DAAC) for May, June, July, September and October with delivered indices and Global
Digital Elevation Model (GTOPO30) with morphometric characteristics (Fig. 1). The seven levels of relief structures were determined with linear sizes ranging from $1.8 \mathrm{~km}$ to $35 \mathrm{~km}$.

At this resolution scale, the association groups on large-scale maps were aggregated to association classes for small-scale interpolation. Subsequently, vector-formatted large-scale maps were reproject from UTM to Geographic coordinate system and converted to the grid format according to the small-scale grid of external variables $(0.008333 \mathrm{dd}$ or $\sim 460 \times 930 \mathrm{~m}$ per pixel). As the result, largescale map was represented by 24642 points in small-scale grid. Interpolations of the small-scale maps of association classes were based on the stepwise discriminant analysis, as was done for large-scale mapping.

\section{RESULTS AND DISCUSSION}

At large scale, we mapped 54 typological units, which characterized 47 association groups of forest and non-forest vegetation types and 7 land cover types of areas without vegetation cover (Table 1). The relative quality of discriminant analysis was $71.5 \%$. The accuracy was over $75 \%$ for 23 units, varied between $50 \%$ and $74 \%$ for 23 units, between $26 \%$ and $49 \%$ for 6 units, and was equal $25 \%$ for 2 units. Large scale vegetation map (Fig. 3) of the model area in the central part of the Kola Peninsula shows that plain areas, as well as the lower and mid-slope areas (200-350 m a.s.l.) are covered by coniferous forests - spruce forests (Picea obovata) with pine (Pinus sylvestris), and birch (Betula pubescens), dwarf shrubs (Empetrum hermaphroditum, Vaccinium spp., Ledum palustre), green mosses (Pleurozium schreberi, Hylocomiun splendens), and lichens (Cetraria islandica, Cladonia spp.). On the plains and mountain valleys (180-250 $\mathrm{m}$ a.s.l.) pine forests (Pinus sylvestris) with birch (Betula pubescens), dwarf shrubs (Vaccinium vitis-idaea, $V$. uliginosum, Empetrum hermaphroditum), green mosses (Pleurozium schreberi, Polytrichum juniperinum) and lichens (Cetraria islandica, Cladonia spp.) are also common. Low-lands, lake basins, and river valleys are covered by marshes. Higher elevations (350 $450 \mathrm{~m}$ a.s.l.) are covered by mountain birch forests and open 
forests (Betula pubescens ssp. czerepanovi) sometimes mixed with spruce (Picea obovata) and pine (Pinus sylvestris), dwarf shrubs (Empetrum hermaphroditum, Betula nana, Calluna vulgaris), green mosses (Pleurozium schreberi, Hylocomiun splendens) and lichens (Cladonia spp., Cetraria islandica, Flavocetraria nivalis).

On the upper slopes and hilltops (400-800 $\mathrm{m}$ a.s.l.), the belt of "warped" birch is replaced by tundra: first, shrubmoss; then, shrub-lichen tundra. Areas of even higher elevation are covered by epilithic lichen communities with small contribution by mosses, growing on the surfaces of stones and rocks substrata, and isolated vascular plants (occasional small fragments of tundra communities) - dwarf shrubs (Silene acaulis, Empetrum hermaphroditum) and herbs (Juncus trifidus, Festuca ovina, Carex bigelowii). Epilithophytic lichen vegetation (Rhizocarpon geographicum, Umbilicaria spp., Arctoparmelia centrifuga) and fruticose lichens (Flavocetraria nivalis, Alectoria ochroleuca, Cetraria islandica), occupy stone outcrops and rocks. Bare rocks and nival areas are found on some mountain ranges at altitude over $1200 \mathrm{~m}$ a.s.l.

Analyses of spatial distribution of plant communities using field data, RSD, DEM, and statistical methods made it possible to depict their composition and structure with consideration of successional status and anthropogenic impacts. Altitudinal gradient of mountain ranges, anthropogenic disturbance, and the natural dynamics of plant communities at different successional stages are the main factors affecting spatial differentiation of vegetation cover in Khibiny model area. Fires and air pollution emissions from smelter are among the types of anthropogenic impacts that prevail in this region (Chernenkova et al. 2011). The cartographic depiction of the anthropogenic succession units depends largely on the scale, and is least challenging for a large-scale map. Understanding information contained in these units allows to assess the intensity of impact. For example, the presence of Spruce forest with birch dwarf shrub-liverwort mosses (Barbilophozia spp., Orthocaulis spp., Lophozia spp.) and sparse lichens (Cetraria islandica, Cladonia spp.) in the vicinity of metallurgical plant may indicate a $10-50$ fold increase in heavy metal content in soils, compared with natural background metal concentrations. Presence of Spruce forest with birch dwarf shrub-corticulous lichens (Cladonia deformis, Trapeliopsis granulosa) may indicate even greater contamination level (e.g., 50-100 fold increase compared with background) (Chernenkova \& Kuperman 1999, Chernenkova et al. 2011). Analysis of the map showed that approximately $23 \%$ of the study area is covered by anthropogenically-modified vegetation.

The 54 mappable units of large-scale map were aggregated to the 28 units for small-scale mapping. The legend for the small-scale map reveals the composition of vegetation types (Table 1), and is analogous with the thematic classes of vegetation map of Europe (Bohn et al. 2004). The legend also reveals the secondary character of forest vegetation, thus takes into account an anthropogenic disturbance of the current vegetation cover in Europe.

The overall relative quality of the discriminant analysis in this case was $52.1 \%$. The accuracy was over $75 \%$ for 6 classes, ranged from $50 \%$ to $74 \%$ for 10 classes, and from $25 \%$ to $49 \%$ for 12 classes.
Transition to smaller scale maps requires a certain degree of generalization in the allocation and depiction of classification units, including adjustments in impact factors and vegetation units.

Analysis of mapping units of vegetation and land cover types at small-scale (Fig. 4 a) revealed that approximately $48 \%$ of total area belongs to forests, including pine forests $-25 \%$ (16\% of which are modified types), spruce forests - $11 \%$ (20\% - modified types), and small-leaved forests $-12 \%$ (26\% - modified types). Barrens cover $3.3 \%$, tundra $-16 \%$, forest-tundra and elfin birch forests $-11 \%$, and the marshes $-17 \%$. Surface water covers approximately - $3.5 \%$ of the territory. Farmlands, settlements, tailings and open pits are about $1 \%$ of total area. Secondary successional communities identified for Fennoscandia at a small scale represent more general types of communities with strongly modified structure and composition due to compounded effects of anthropogenic factors (Table 1). Overall, approximately $19 \%$ of the forest cover within this area is covered by such type of derivative forests.

There is an insufficient availability of small-scale maps that are based on satellite data for northwestern Europe. A single map is available for area of northern parts of Finland, Sweden and adjacent territories of Norway and Kola Peninsula (Johansen \& Karlsen 2008). Based on the visual comparison, the map developed in the present project is in good agreement with the map developed by the Norwegian researches. Small differences may be related to direct vegetation classifications that underlie each map product.

Substantial differences were revealed when comparing maps developed in the present study with the previously developed map of natural vegetation of Europe (Bohn et al. 2004) (Fig. 4 a, b). A clear disparity is manifested not only in the more precise contours of all selected groups, but also in the distribution of pine and spruce forests in Karelia, Finland and Sweden. Such differences may be related to different classification approaches in the national classification systems used in different countries. Based on the dynamic classification, originally developed by Cajander (1926), spruce forests represent potentially climax communities in the considerable part of Finland and Sweden. Our present map shows widely spread pine forests derived from spruce forests in Fennoscandia.

\section{CONCLUSIONS}

The present studies developed the methodology and methods for cartographic modeling of vegetation at different spatial scales. Results of the study allowed to start a comparative analysis of the boreal forest ecosystem diversity at the regional level in natural regions of Eastern European part of Russia. Empirical data on the composition and structure of forest communities of Northern Kola Peninsula allowed us to assess coenotic diversity, identify trends in diversity changes, and evaluate the role of different factors in differentiation of forest cover, all in relation with both natural characteristics of the territory, as well as with human impact.

In our study a method for constructing large- and smallscale maps of vegetation cover was affirmed. This method 
is based on analysis of the characteristics of vegetation obtained by ground surveys, remote sensing data, and morphometric parameters of the relief. This investigation also revealed spatially explicit patterns in structure of forest diversity in the model regions. An assessment of coenotic diversity and the development of maps for different regions were accomplished using consistent approach to vegetation classification. The proposed methodology for describing and mapping vegetation types at different spatial scales has proven to be effective because it is based on uniform hierarchical classification. The integrated hierarchical approach was successful in mapping vegetation in northern Alaska (Walker 1999), Italian landscapes (Blasi et al. 2000, 2011), Canada (Saucier et al. 2013), natural vegetation of Europe (Bohn et al. 2004). In addition to the typological diversity of forests, map developed in the present project reveals ecological specificity and the dynamics of forest communities in relation to their disturbance and successional stage.

Regional maps developed in this investigation can be used for further mapping and synthesis of Eastern European regional components of the country with the aim of developing small-scale maps of the present-day diversity of boreal and hemi-boreal forests. This map depicts the most common forest types (mountain birch forests and open forests, spruce and pine forests), as well as their derivative modifications, including characterization of the dominant species of ground cover.

Results of the analyses of the spatial diversity of Eastern European boreal forests were harmonized with the typological units of vegetation classification and phytogeographical subdivisions of the boreal forest biome within the framework of international project developing circum-boreal vegetation maps (Circum-Boreal Vegetation Mapping, CBVM).

\section{ACKNOWLEGMENTS}

We are grateful to N.E. Koroleva and E.V. Basova for participation in collection of botanical data and their classification, E.A. Ignatova and E.A. Borovichev for moss collection identification, A.V. Melehin for lichen collection identification. This study was supported by the Russian Foundation for Basic Research (grants 07-04-01743, 11-0401093, 14-04-98810 and 14-27-00065).

\section{LITTERATURE CITED}

Bartalev, S.A., A.S. Belward, D.V. Ershov \& A.S. Isaev 2003. A new SPOT-4 VEGETATION derived land cover map of Northern Eurasia. International Journal of Remote sensing 24(9):1977-1982.

Bartalev, S.A., V.A. Egorov, D.V. Ershov, A.S. Isaev, E.F. Lupian, D.E. Plotnikov \& I.A. Uvarov 2011. Satellite mapping of vegetation cover over Russia using data from spectroradiometer MODIS. Sovremennye problemy distantsionnogo zondirovaniya Zemli iz. kosmosa 8(4):285-302 (in Russian). ББарталев С.А., Егоров В.А., Ершов А.В., Исаев А.С., Аупян Е.А., ПАотников А.Е., Уваров И.А. 2011. Спутниковое картографирование растительного покрова России по данным спектрорадиометра MODIS // Современные проблемы Аистанционного зондирования Земли из космоса. Т. 8, № 4. С. 285-302].
Belov, A.V., S.A. Gribova \& T.V. Kotova (eds.) 1990. Map "Vegetation of the USSR" (scale 1:4 000 000). Explanatory text and map legend. GUGK USSR, Moscow (in Russian). [Карта «Растительность СССР». 1990. Масштаб 1:4 000000 / Отв. ред. А.В. Белов, С.А. Грибова, Т.В. Котова. Пояснительный текст и мегенда карты. М.: ГУГК СССР].

Blasi, C., M.L. Carranza, R. Frondoni \& L. Rosati 2000. Ecosystem classification and mapping: a proposal for Italian landscapes. Applied Vegetation Science 3: 233-242.

Blasi, C., G. Capotorti, R. Frondoni, D. Guida, B. Mollo, D. Smiraglia \& L. Zavattero 2011. Vegetation science and the ecoregional approach: a proposal for the ecological land classification of Italy. Fitosociologia 48(2): 75-82.

Bohn, U., G. Gollub, C. Hettwer, H. Weber, Z. Neuhäuslová, T. Raus \& H. Weber 2004. Karte der natürlichen Vegetation Europas. Mapofthenaturalvegetationof Europe (scale 1 : 2500 000). Federal Agency for Nature Conservation. Bonn.

Cajander, A.K. 1926. The theory of forest types. Acta Forestalia Fennica 29(3):1-108.

Cherepanov, S.K. 1995. Vascular plants of Russia and neighboring countries (in boundaries of former USSR). S.-Pb, $992 \mathrm{p}$. [Черепанов С.К. 1995. Сосудистые растения России и сопредельных государств (в пределах бывшего СССР). С.-Пб.: Мир и семья, 992 с.].

Chernenkova, T.V. \& R.G. Kuperman 1999. Changes in the spruce forest communities along a heavy metal deposition gradient on Kola peninsula. Water, Air and Soil Pollution 111:187-200.

Chernenkova, T.V. \& D.N. Koslov 2009. Dynamic of Moscow region forest using satellite data. Zemlya iz kosmosa 1:22-26 (in Russian). [Черненькова Т.В., КозАов А.Н. 2009. Аинамика месов Подмосковья по материалам космической съемки // Земля из космоса. № 1. С. 22-26].

Chernenkova, T.V., R.R. Kabirov \& E.V. Basova 2011. Regeneration successions of northern taiga spruce forests under reduction of aerotechnogenic impact. Contemporary Problems of Ecology 4(7):742-757.

Chernenkova, T.V., M.Yu. Puzachenko, O.V. Morozova, G.N. Ogureeva, N.E. Koroleva, \& N.G. Kadetov 2012. Approaches to the evaluation of spatial variability of Eastern european boreal forest using field and remote sensing data. Iqvestiya Samarskogo nauchnogo centra RAN 14 (1/6): 1652-1655 (in Russian with English abstract). [Черненькова Т.В., Пузаченко М.Ю., Морозова О.В., Огуреева Г.Н., Королева Н.Е., Кадетов Н.Г. 2012. Подходы к оценке пространственной вариабельности восточноевропейских бореальных месов на основе наземных и Аистанционных данных // Известия Самарского научного центра РАН. Т. 14, № 1(6). С. 1652-1655.]

ENVI - program complex Environment for visualizing images, available at: http://www.exelisvis.com/ProductsServices/ENVIProducts.aspx.

Gribova, S.A \& R. Neuhäusl (eds.) 1989. Map of Reconstructed vegetation of Central and Eastern Europe (scale $1: 2500$ 000). UNESCO (in Russian). [Карта восстанов енной растительности Центральной и Восточной Европы (масштаб 1:2 500 000) / под реА. С.А. Грибовой и Р. Нейхейсла. ЮНЕСКО, 1989].

Ignatov, M.S., O.M. Afonina \& E.A. Ignatova 2006. Checklist of mosses of East Europe and North Asia. Arctoa: 15:1-130.

Johansen, B.E. \& S.R. Karlsen 2008. Vegetation Mapping of Norway and Northern Scandinavia Using Satellite Data. In: Proceedings of the Fourth International Conservation 
of Arctic Flora and Fauna (CAFF) Flora Group Workshop, 15-18 May 2007, Tórshavn, Faroe Islands. CAFF Technical Report N 15 (S.S. Talbot, ed.), pp. 89-90, Akureyri.

Kim, J.O., C.W. Mueller, W.R. Klekka, M.S. Aldenderfer \& R.K. Blashfield 1989. Factorial, discriminant and cluster analysis. Translated to Russian. Finansy and Statistika, Moscow, 215 рр. [Ким Аж.О., Мюлиер Ч.У., Клекка У.Р., Олленлерфер М.С., БАэшфильА Р.К. Факторный, Аискриминантный и кластерный анализ: Пер. с англ. / под ред. И.С. Енюкова. М.: Финансы и статистика, 1989. 215 c.].

Konstantinova, N.A. \& V.A. Bakalin 2009. Check-list of liverworts (Marchantiophyta) of Russia. Arctoa 18:1-64.

Kozlov, D.N., M.Yu. Puzachenko, M.N. Fedyaeva \& Yu.G. Puzachenko 2008. To display spatial variation of properties of landscape cover based on distance information and digital elevation models. Iqvestiya RAN, Seriya geographicheskaya 4: 112-124 (in Russian with English summary). [КозАов A.H., Пузаченко М.Ю., Федяева М.Н., Пузаченко Ю.Г. 2008. Отображение пространственного варьирования свойств Аандшафтного покрова на основе дистанционной информации и цифровой модели рельефа // Известия РАН, серия географическая. № 4. С. 112-124].

Kravtzova, V.I. 2013. On interpretation of northern forests on cosmos images of super-high resolution. Lesovedenie 1:56-65 (in Russian with English summary). [Кравцова В.И. 2013. Особенности Аешифрирования север-

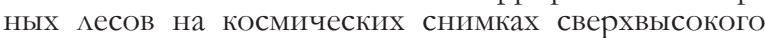
разрешения // Аесоведение. № 1. С. 56-65].

Lavrenko, E.M. 1968. The regular tasks of the geography study of vegetation cover due to the zoning of the USSR. The main problems of modern geobotany. Leningrad: 45-69 (in Russian). [Аавренко E.M. 1968. Об очередных задачах изучения географии растительного покрова в связи с ботаническим районированием СССР // Основные проблемы современной геоботаники. $\Lambda$. С. 45-69.]

Lavrenko, E.M. (ed.) 1976. Map of the vegetation of European part of the former USSR and the Transcaucasia (scale 1:2 500 000). Leningrad (in Russian). [Карта растительности европейской части СССР и Закавказья. 1976. Масштаб 1:2 500000 / под ред. Е.М. Аавренко. АенинграА].

McRober, R.E. 2006. A model-based approach to estimating forest area. Remote Sensing of Environment 103:56-66.

NASA LP DAAC - NASA Land Processes Distributed Active Archive Center, available at: http://lpdaac.usgs.gov/ products/modis_products_table/mcd $43 \mathrm{~b} 4$.

Puzachenko, M.Yu \& T.V. Chernenkova 2012. Assessment of the vegetation cover conditions for the central part of the Murmansk region based on field and remote sensing data. Geography, Environment, Sustainability 5(3):4-13.

Puzachenko, M.Yu., T.V. Chernenkova \& E.V. Basova 2012. Analysis of natural and anthropogenic differentiation of the vegetation cover for the central part of the Murmansk region. In: Biodiversity and dynamic of forest ecosystems of Russia, vol. 1 (A.S. Isaev, ed.), pp. 371-382, KMK Scientific Press, Moscow (in Russian). [Пузаченко M.Ю., Черненькова Т.В., Басова Е.В. 2012. Анализ природноантропогенной неоднородности растительного покрова центральной части Мурманской области // Разнообразие и динамика лесных экосистем России. Т. 1 / под ред. А. С. Исаева. Москва: Товарищество научных изАаний КМК. С. 371-382].

Puzachenko, Yu.G., I.A. Onufrenya, G.M. Aleshchenko 2002. Analysis of the hierarchical organization of relief.
Izvestiya $\mathrm{RAN}$, seriya geograficheskaya 4:29-38 (in Russian with English summury). [Пузаченко Ю.Г., Онуфреня И.А., Алещенко Г.М. 2002. Анализ иерархической организации рельефа // Известия РАН, серия географическая. № 4. С. 29-38].

Puzachenko, Yu.G. 2004. Mathematical methods in ecological and geographical studies. ACADEMA, Moscow, 416 pp. (in Russian). [Пузаченко Ю.Г. 2004. Математические методы в экологических и географических исследованиях. Москва: ACADEMA. 416 с.].

Puzachenko, Yu.G., I.P. Kotlov \& R.B. Sandlerskij 2014. Analysis of the landscape cover changes according to the distance multi-spectral information in the Central FOrest Reserve. Iqvestiya RAN, seriya geograficheskaya 3: 5-18 (in Russian with English summury). ППзаченко Ю.Г., Котлов И.П., Сандмерский Р.Б. 2014. Анализ изменений мандшафтного покрова по Аанным мультиспектральной Аистанционной информации в Центрально-лесном Заповеднике // Известия РАН, серия географическая. № 3. С. 5-18].

Rodwell J., J. Schaminée, L. Mucina, S. Pignatti, J. Dring \& D. Moss 2002. The diversity of European vegetation. An overview of phytosociological alliances and their relationships to EUNIS habitat. Landbouw, Naturbeheer en visserij / European Environment Agency., Wageningen.

Roelofsen, H.D., L. Kooistra, P.M. van Bodegom, J. Verrelst, J. Krol \& J.-P.M. Witte 2014. Mapping a priori defined plant associations using remotely sensed vegetation characteristics. Remote Sensing of Environment 140:639-651.

Rysin, L.P. \& L.I. Savelyeva 2007. Inventories of forest types and types of forest biocoenoses. KMK Scientific Press, Moscow. 144 pp. (in Russian). [Рысин А.П., Савельева А.И. 2007. Кацастры типов Аеса и типов месных биогеоценозов. М.: Товарищество научных изданий КМК. 144 с.].

Santesson, R., R. Moberg, A. Nordin, T. Tonsberg \& O. Vitikainen 2004. Lichen-forming and lichenicolous fungi of Fennoscandia. Uppsala, 359 pp.

Saucier, J.-P., K. Baldwin, W.B. Meades, D. Meidinger, W. Mackenzie, A. Robitaille \& P. Uligh 2013. The CBVM legend and its application for mapping the boreal vegetation of Canada. CAFF Proceeding Series Report. Nr. 9, February 2013 , pp. 16-22.

Sochava, V.B. 1964. The model of new world vegetation map. In: Geobotanicheskoe Kartografirovanie, pp. 3-16, Moscow-Leningrad, Nauka (in Russian). [Сочава В.Б. 1964. Макет новой карты растительности мира // Геоботаническое картографирование. М.-. .: Наука. С. 3-16].

Tomppo, E., H. Olsson, G. Ståhl, M. Nilsson, O. Hagner \& M. Katila 2008. Combining national forest inventory field plots and remote sensing data for forest databases. Remote Sensing of Environment 112:1982-1999.

Walker, D.A. 1999. An integrated vegetation mapping approach for northern Alaska (scale 1:4 000 000). International Journal of Remote sensing 20(15):2895-2920.

Zaugolnova, L.B. \& O.V. Morozova 2006. Typology and classification of European Russia's forests: method approaches and possibility of realization. Lesovedenie 1:115 (in Russian with English summary). [Заугольнова А.Б., Морозова О.В. 2006. Типология и классификация месов Европейской России: методические подходы и возможности их реализации // Аесоведение. № 1. С. $1-15]$. 